\title{
ISM / RADIO MATERIAL INTERACTION MODELING
}

\author{
P. FERRUIT, L. BINETTE AND E. PECONTAL \\ CRAL Observatoire de Lyon - UMR142 \\ 9, av. Charles André - 69561 Saint-Genis-Laval Cedex, France
}

With the outcoming of high angular resolution imaging and 3D spectrographic facilities (HST, TIGER-OASIS [1] at the CFH Telescope...), the need of spatially resolved theoretical models of the kinematic interaction of radio material with the ISM (or the IGM for radio-galaxies) is increasing dramatically. In order to fill the gap between pure hydrodynamical models (3D approach, but very poor atomic physic) and photoionisation/shocks ones (planar description, full account for atomic physic processes), we have started to develop 2D or 3D hydrodynamical models including a good description of atomic physic processes (using photoionisation routines from the code MAPPINGS [2]).

We present here the first results of a bowshock model based on that of Taylor et al. [3]. We have improved their description in two main ways :

- by using an adaptive step size when following a cooling particle. This is particularly important in the "catastrophic" cooling zone where the gas temperature drops very rapidly to $10^{4} \mathrm{~K}$ (see Fig. 1).

- by using MAPPINGS for the computation of the ionization equilibrium and emission line fluxes.

When comparing our results with that of Taylor et al., we have found a large discrepancy between our [OIII] fraction and theirs. This is likely due to charge transfer reactions that they did not take into account (see Fig. 2). It points out the importance of a good atomic physic description.

We have also explored the effect of changing the ionization parameter (by varying the nuclear source flux). This highlights the major role played by photoionization even in a shock based model! As the bulk of the emission originates from the photoionised zone, this puts in question the use of line ratios as shock diagnostics.

Follow up work will aim at introducing out-of-equilibrium processes affecting ionisation states, and at studying in more details the relative contribution of shocks and photoionisation, to the emission. 

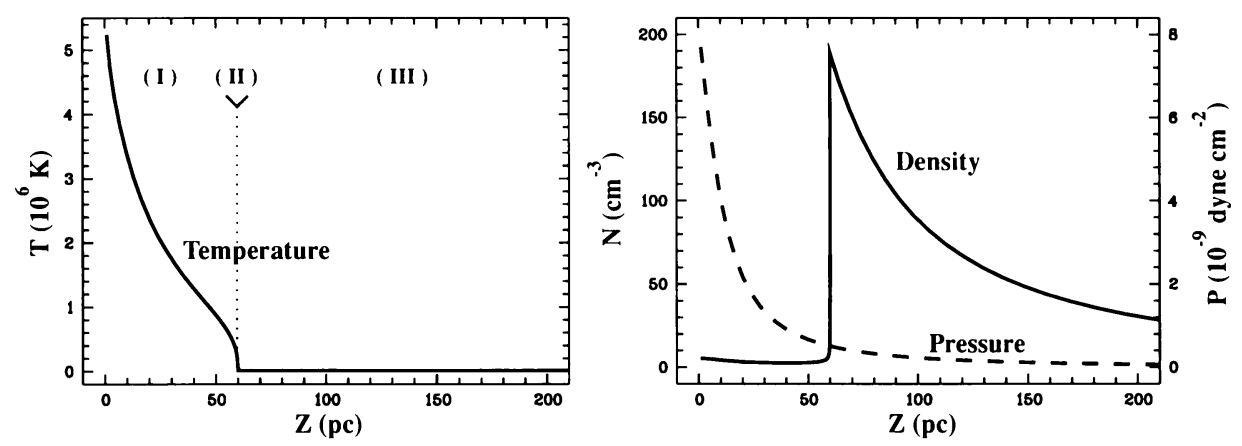

Figure 1. Evolution of a particle that entered the bowshock at $\mathrm{Z}=1$ pc. Model parameters : shock velocity $700 \mathrm{~km} \mathrm{~s}^{-1}$; ISM hydrogen number density $1 \mathrm{~cm}^{-3}$; ISM ionization parameter $\mathrm{U}=10^{-3}$; power law (spectral index of 1.4) used for the central nuclear source; geometrical parameters $D_{Z}=40 \mathrm{pc}, \mathrm{B}=0.6$ (E model of Taylor et al. with lower ionization parameter). Zone $I$ : the pressure decreases slightly faster than the temperature, inducing a slow density decrease. Zone II : catastrophic cooling, the temperature goes to $10^{4} \mathrm{~K}$ in a few parsec inducing a strong density enhancement (constant pressure). Zone III : the gas has cooled, the density decreases slowly with the pressure.
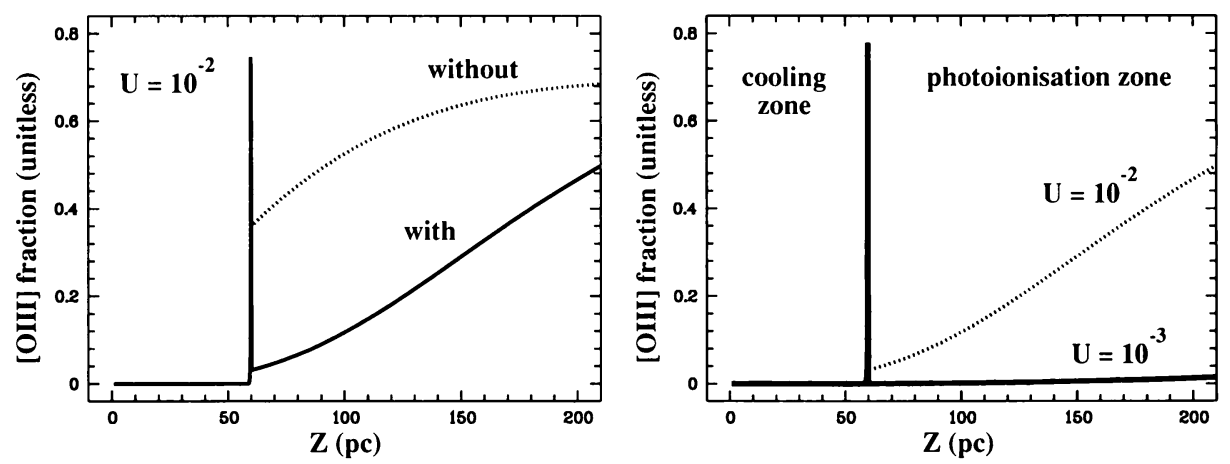

Figure 2. Left : [OIII] fraction as a function of $\mathrm{Z}$ with and without including charge transfer reactions in the modelisation. Right : [OIII] fraction evolution for two different ISM ionisation parameters. In the cooling zone, the temperature is too high for [OIII] to exist. In the photoionisation zone, its fraction grows slowly as the ionisation parameter $\mathrm{U}$ increases (the density decreases with $\mathrm{Z}$ ).

\section{References}

[1] R. Bacon, et al., 1995. A\& A Supp., 113, 347.

[2] L. Binette, 1982. Ph.D. thesis, Australian National. University, Canberra

[3] D. Taylor, J.E. Dyson, D.J. Axon, 1992. MNRAS, 255, 351. 